\title{
RECURSIVE FILTERS FOR PARTIALLY OBSERVABLE FINITE MARKOV CHAINS
}

\author{
JAMES LEDOUX,* Centre de Mathématiques INSA and IRMAR, Rennes
}

\begin{abstract}
In this note, we consider discrete-time finite Markov chains and assume that they are only partly observed. We obtain finite-dimensional normalized filters for basic statistics associated with such processes. Recursive equations for these filters are derived by means of simple computations involving conditional expectations. An application to the estimation of parameters of the so-called discrete-time batch Markovian arrival process is outlined.
\end{abstract}

Keywords: Time-inhomogeneous Markov chain; hidden Markov chain; D-BMAP; EM-algorithm

2000 Mathematics Subject Classification: Primary 60J10; 93E11

\section{Introduction}

Let $(X, Y):=\left(X_{t}, Y_{t}\right)_{t \geq 0}$ be a bivariate discrete-time finite (inhomogeneous) Markov chain. Only the second component, $Y$, is supposed to be observable: $Y$ is often called the observation process and $X$ the 'hidden' process. The special case of hidden Markov models is well known. A hidden Markov chain is a bivariate Markov chain for which the hidden component is also a Markov chain. This class of Markovian model has proved to be useful in many areas of probabilistic modeling, including speech processing (e.g. see [15] and the references therein).

At time $t$, the available data on $(X, Y)$ consists of the sequence of values $Y_{0}, \ldots, Y_{t}$. A standard problem is to find from the data the best estimate, at time $t$, of quantities related to the unobserved process $X$. Such a problem is often referred to as a filtering problem. In this paper, finite-dimensional filters for basic statistics related to $X$ and $Y$ are obtained in a recursive form. Our only assumption is that $(X, Y)$ is a finite Markov chain. The derivation of our main result is based on basic computations involving conditional expectations. Specializing our result to time-homogeneous dependent hidden Markov chains, we find a renormalized form of the Zakaï filters obtained by Elliott in [7] and [9]. The change-of-measure technique used by Elliott has the advantage of providing a unified framework for the computation of filters for hidden Markov chains. In particular, it works for hidden Markov chains continuous in both time and space [9]. Note that, to be suitable, such a change of measure must preserve the Markov property of the hidden component $X$. In the context of discrete time and space, we believe our approach to be more direct and elementary.

In this paper, the number of 'states' of $X$ is assumed to be known. This is adequate, for example, in speech recognition, where the hidden states are the elements of a finite alphabet, and in architecture-based software reliability modeling, where the hidden states are the modules of a piece of software. However, in many applications, this is not the case. A generic situation

Received 12 October 2004; revision received 4 January 2005.

* Postal address: Centre de Mathématiques INSA, 20 avenue des Buttes de Coësmes, CS 14315, 35043 Rennes cedex, France. Email address: james.ledoux@insa-rennes.fr 
is when a partially observed model is used as a statistical model for fitting to empirical timeseries. Estimation of the number of 'hidden states' is known to be a hard problem. We do not address this fundamental issue here. In the context of hidden Markov chains, the so-called 'order estimation problem' was surveyed in [10, Section VIII]. Recent progress in this direction was reported in [11] and [5]. We refer the reader to these papers and the references therein for details.

The paper is organized as follows. In Section 2, we state our main result. Then, in Section 3, we specialize the result to time-inhomogeneous dependent hidden Markov chains. We also mention the connection of our result with the so-called 'innovation approach' for filtering. In Section 4, we briefly describe parameter estimation for the discrete-time counterpart of the batch Markovian arrival process. Here, a filter-based form of the expectation-maximization (EM-)algorithm is used. Section 5 is devoted to the proof of our main result. Finally, we present our conclusions in Section 6.

\subsection{Main notation}

Throughout, we will use the following notation.

- By convention, vectors are column vectors. Row vectors are denoted by means of the transpose operator $(\cdot)^{\top}$. The $(i, j)$ th component of a generic matrix $\boldsymbol{A}$ is denoted by $A(i, j)$.

- $\mathbf{1}$ and $\mathbf{0}$ are vectors in which each entry is equal to 1 or 0 , respectively. Their dimensions are defined by the context.

- $\boldsymbol{e}_{i}$ and $\boldsymbol{f}_{j}$ are the $i$ th vector of the canonical basis of $\mathbb{R}^{n}$ and the $j$ th vector of the canonical basis of $\mathbb{R}^{m}$, respectively.

- For any vector (or scalar) $\boldsymbol{a}$ and scalar $b \in \mathbb{R}$,

$$
\left(\frac{\boldsymbol{a}}{b}\right)^{+}= \begin{cases}\boldsymbol{a} / b, & b \neq 0 \\ \mathbf{0}, & b=0\end{cases}
$$

Note that, for a scalar $b,(b / b)^{+}$is the indicator function of the set $\{b \neq 0\}$.

\section{Recursive filters for partially observable finite Markov chains}

In this section, $(X, Y)$ is only assumed to be a finite Markov chain. The state space of $(X, Y)$ is assumed to be the finite set $E \times F$, where $E:=\left\{\boldsymbol{e}_{i}, i=1, \ldots, n\right\}$ and $F:=\left\{\boldsymbol{f}_{k}, k=\right.$ $1, \ldots, m\}$. Note that, with our conventions,

$$
1_{\left\{X_{t}=e_{i}\right\}}=\left\langle X_{t}, \boldsymbol{e}_{i}\right\rangle=\boldsymbol{e}_{i}^{\top} X_{t}, \quad X_{t}=\sum_{i=1}^{n}\left\langle X_{t}, \boldsymbol{e}_{i}\right\rangle \boldsymbol{e}_{i}, \quad \mathbf{1}^{\top} X_{t}=1,
$$

where $1_{\{\cdot\}}$ is the indicator function of the set $\{\cdot\}$ and $\langle\cdot, \cdot\rangle$ denotes the usual Euclidean scalar product. The corresponding relations also hold for the observed process $Y$. All processes are supposed to be defined on the same probability space $(\Omega, \mathcal{F}, \mathrm{P})$. We denote by $\mathbb{F}^{X}=\left(\mathbb{F}_{t}^{X}\right)_{t \geq 0}$, $\mathbb{F}^{Y}=\left(\mathbb{F}_{t}^{Y}\right)_{t \geq 0}$, and $\mathbb{F}^{X, Y}=\left(\mathbb{F}_{t}^{X, Y}\right)_{t \geq 0}$ the internal filtrations of processes $X, Y$, and $(X, Y)$, respectively. That is,

$$
\mathbb{F}_{t}^{X}:=\sigma\left(X_{s}, s \leq t\right), \quad \mathbb{F}_{t}^{Y}:=\sigma\left(Y_{s}, s \leq t\right), \quad \mathbb{F}_{t}^{X, Y}:=\sigma\left(X_{s}, Y_{s}, s \leq t\right) .
$$


These filtrations are assumed to be complete, that is, every $\sigma$-algebra contains all the sets of $\mathcal{F}$ of P-probability 0.

In this paper, we deal with the following statistics of the partially observed Markov chain, for any time $t \geq 1$ and $i, j=1, \ldots, n$ :

- the number of jumps of $X$ from $\boldsymbol{e}_{i}$ to $\boldsymbol{e}_{j}$ up to time $t$, i.e.

$$
N_{t}^{j i}:=\sum_{l=1}^{t}\left\langle X_{l}, \boldsymbol{e}_{j}\right\rangle\left\langle X_{l-1}, \boldsymbol{e}_{i}\right\rangle
$$

- the number of visits of $X$ to state $\boldsymbol{e}_{i}$ up to time $t-1$, i.e.

$$
\mathcal{O}_{t}^{(i)}:=\sum_{l=1}^{t}\left\langle X_{l-1}, \boldsymbol{e}_{i}\right\rangle
$$

- the number of times, up to time $t$, that $(X, Y)$ jumps to state $\left(\boldsymbol{f}_{k}, \boldsymbol{e}_{j}\right)$, given that $X$ is in state $\boldsymbol{e}_{i}$ at the previous time, i.e.

$$
\mathcal{L}_{t}^{k, j i}:=\sum_{l=1}^{t}\left\langle Y_{l}, \boldsymbol{f}_{k}\right\rangle\left\langle X_{l}, \boldsymbol{e}_{j}\right\rangle\left\langle X_{l-1}, \boldsymbol{e}_{i}\right\rangle, \quad k=1, \ldots, m .
$$

We are interested in the quadratic mean estimates for these scalar random variables, from the data $\left(Y_{0}, \ldots, Y_{t}\right)$ :

$$
\widehat{N}^{j i}{ }_{t}:=\mathrm{E}\left[N_{t}^{j i} \mid \mathbb{F}_{t}^{Y}\right], \quad \widehat{\mathcal{O}^{(i)}}{ }_{t}:=\mathrm{E}\left[\mathcal{O}_{t}^{(i)} \mid \mathbb{F}_{t}^{Y}\right], \quad \text { and } \quad \widehat{\mathcal{L}^{k, j i}}{ }_{t}:=\mathrm{E}\left[\mathcal{L}_{t}^{k, j i} \mid \mathbb{F}_{t}^{Y}\right] .
$$

In fact, we find that $\hat{X}_{t}:=\mathrm{E}\left[X_{t} \mid \mathbb{F}_{t}^{Y}\right]$ and the filters defined by

$$
\begin{aligned}
{\widehat{N^{j i} X}}_{t} & :=\mathrm{E}\left[N_{t}^{j i} X_{t} \mid \mathbb{F}_{t}^{Y}\right], \\
\widehat{\mathcal{O}}^{(i)} X_{t} & :=\mathrm{E}\left[\mathcal{O}_{t}^{(i)} X_{t} \mid \mathbb{F}_{t}^{Y}\right], \\
\widehat{\mathcal{L}^{k, j i} X_{t}}: & =\mathrm{E}\left[\mathcal{L}_{t}^{k, j i} X_{t} \mid \mathbb{F}_{t}^{Y}\right],
\end{aligned}
$$

for any $t \geq 0$, each satisfies a recursive equation. Since $\mathbf{1}^{\top} X_{t}=1$, the filters in (2.4) can be written

$$
{\widehat{N{ }^{j i}}}_{t}=\mathbf{1}^{\top}{\widehat{N^{j i} X}}_{t}, \quad \widehat{\mathcal{O}}^{(i)}{ }_{t}=\mathbf{1}^{\top} \widehat{\mathcal{O}}^{(i)} X_{t}, \quad \text { and } \quad{\widehat{\mathcal{L}^{k, j i}}}_{t}=\mathbf{1}^{\top}{\widehat{\mathcal{L}^{k, j i}}}_{t} .
$$

For any $t \geq 0$, we define the $n \times m$ random matrix $S\left(t ; X_{t}, Y_{t}\right)$ as follows: for any $j=$ $1, \ldots, n$ and $k=1, \ldots, m$, its $(j, k)$ th entry is

$$
\begin{aligned}
S\left(t ; X_{t}, Y_{t}\right)(j, k): & =\mathrm{E}\left[\left\langle X_{t+1}, \boldsymbol{e}_{j}\right\rangle\left\langle Y_{t+1}, \boldsymbol{f}_{k}\right\rangle \mid X_{t}, Y_{t}\right] \\
& =\mathrm{P}\left(\left(X_{t+1}, Y_{t+1}\right)=\left(\boldsymbol{e}_{j}, \boldsymbol{f}_{k}\right) \mid X_{t}, Y_{t}\right) .
\end{aligned}
$$

Then the Markov property of $(X, Y)$, the equality

$$
X_{t+1} Y_{t+1}^{\top}=\sum_{j=1}^{n} \sum_{k=1}^{m}\left\langle X_{t+1}, \boldsymbol{e}_{j}\right\rangle\left\langle Y_{t+1}, \boldsymbol{f}_{k}\right\rangle \boldsymbol{e}_{j} \boldsymbol{f}_{k}^{\top},
$$


and (2.5) allow us to write

$$
\begin{aligned}
\mathrm{E}\left[X_{t+1} Y_{t+1}^{\top} \mid \mathbb{F}_{t}^{X, Y}\right] & =\mathrm{E}\left[X_{t+1} Y_{t+1}^{\top} \mid X_{t}, Y_{t}\right] \\
& =\sum_{j=1}^{n} \sum_{k=1}^{m} \mathrm{E}\left[\left\langle X_{t+1}, \boldsymbol{e}_{j}\right\rangle\left\langle Y_{t+1}, \boldsymbol{f}_{k}\right\rangle \mid X_{t}, Y_{t}\right] \boldsymbol{e}_{j} \boldsymbol{f}_{k}^{\top} \\
& =\boldsymbol{S}\left(t ; X_{t}, Y_{t}\right), \quad t \geq 0 .
\end{aligned}
$$

Since $\mathbf{1}^{\top} X_{t}=1$, we deduce from (2.6) that, for every $t \geq 0$,

$$
\mathrm{E}\left[Y_{t+1} \mid \mathbb{F}_{t}^{X, Y}\right]=\mathrm{E}\left[Y_{t+1} \mid X_{t}, Y_{t}\right]=S\left(t ; X_{t}, Y_{t}\right)^{\top} \mathbf{1}
$$

Let $\left(U_{t}\right)_{t \geq 0}$ be any sequence of integrable random variables. For any $t$, set $\hat{U}_{t}:=\mathrm{E}\left[U_{t} \mid \mathbb{F}_{t}^{Y}\right]$. In an abuse of notation, we denote by $\boldsymbol{S}\left(t ; \hat{U}_{t}, Y_{t}\right)$ the $n \times m$ random matrix

$$
\boldsymbol{S}\left(t ; \hat{U}_{t}, Y_{t}\right):=\sum_{i=1}^{n} \boldsymbol{S}\left(t ; \boldsymbol{e}_{i}, Y_{t}\right)\left\langle\hat{U}_{t}, \boldsymbol{e}_{i}\right\rangle
$$

Since $\boldsymbol{S}\left(t ; X_{t}, Y_{t}\right)=\sum_{i=1}^{n} \boldsymbol{S}\left(t ; \boldsymbol{e}_{i}, Y_{t}\right)\left\langle X_{t}, \boldsymbol{e}_{i}\right\rangle$ and $\mathrm{E}\left[\left\langle X_{t}, \boldsymbol{e}_{i}\right\rangle \mid \mathbb{F}_{t}^{Y}\right]=\left\langle\mathrm{E}\left[X_{t} \mid \mathbb{F}_{t}^{Y}\right], \boldsymbol{e}_{i}\right\rangle$, the random matrix $S\left(t ; \hat{X}_{t}, Y_{t}\right)$ is a conditional expectation:

$$
\boldsymbol{S}\left(t ; \hat{X}_{t}, Y_{t}\right)=\mathrm{E}\left[\boldsymbol{S}\left(t ; X_{t}, Y_{t}\right) \mid \mathbb{F}_{t}^{Y}\right] .
$$

Theorem 2.1. 1. (Estimator for the state.) For $k=1, \ldots, m$, let $\boldsymbol{p}_{k}$ be the $n$-dimensional vector defined by $p_{k}(i):=\mathrm{P}\left(X_{0}=\boldsymbol{e}_{i}, Y_{0}=\boldsymbol{f}_{k}\right), i=1, \ldots, n$. For any $t \geq 0$, we have

$$
\hat{X}_{0}=\sum_{k=1}^{m}\left(\frac{p_{k}}{\mathbf{1}^{\top} p_{k}}\right)^{+}\left\langle Y_{0}, \boldsymbol{f}_{k}\right\rangle, \quad \hat{X}_{t+1}=\sum_{k=1}^{m}\left(\frac{\boldsymbol{S}\left(t ; \hat{X}_{t}, Y_{t}\right) \boldsymbol{f}_{k}}{\mathbf{1}^{\top} \boldsymbol{S}\left(t ; \hat{X}_{t}, Y_{t}\right) \boldsymbol{f}_{k}}\right)^{+}\left\langle Y_{t+1}, \boldsymbol{f}_{k}\right\rangle .
$$

2. (Estimator for the number of visits to $\boldsymbol{e}_{i}$.) $\operatorname{Set} \mathcal{O}_{0}^{(i)}:=0$ and, for $t \geq 1$, define $\mathcal{O}_{t}^{(i)}$ according to (2.2). We have $\widehat{\mathcal{O}}^{(i)} X_{0}=0$ and, for any $t \geq 0$,

$$
{\widehat{\mathcal{O}^{(i)} X}}_{t+1}=\sum_{k=1}^{m}\left(\frac{\boldsymbol{S}\left(t ; \widehat{\mathcal{O}}(i)_{t}, Y_{t}\right) \boldsymbol{f}_{k}+\boldsymbol{S}\left(t ; \boldsymbol{e}_{i}, Y_{t}\right) \boldsymbol{f}_{k}\left\langle\hat{X}_{t}, \boldsymbol{e}_{i}\right\rangle}{\mathbf{1}^{\top} \boldsymbol{S}\left(t ; \hat{X}_{t}, Y_{t}\right) \boldsymbol{f}_{k}}\left\langle Y_{t+1}, \boldsymbol{f}_{k}\right\rangle .\right.
$$

3. (Estimator for the number of jumps of $X$ from $\boldsymbol{e}_{i}$ to $\boldsymbol{e}_{j}$.) Set $N_{0}^{j i}=0$ and, for $t \geq 1$, define $N_{t}^{j i}$ according to (2.1). We have $\widehat{N}^{j i} X_{0}=0$ and, for any $t \geq 0$,

$$
{\widehat{N^{j i} X_{t+1}}}_{t}=\sum_{k=1}^{m}\left(\frac{\boldsymbol{S}\left(t ;{\widehat{N{ }^{j i} X}}_{t}, Y_{t}\right) \boldsymbol{f}_{k}+\boldsymbol{e}_{j}^{\top} \boldsymbol{S}\left(t ; \boldsymbol{e}_{i}, Y_{t}\right) \boldsymbol{f}_{k}\left\langle\hat{X}_{t}, \boldsymbol{e}_{i}\right\rangle \boldsymbol{e}_{j}}{\boldsymbol{1}^{\top} \boldsymbol{S}\left(t ; \hat{X}_{t}, Y_{t}\right) \boldsymbol{f}_{k}}\right)^{+}\left\langle Y_{t+1}, \boldsymbol{f}_{k}\right\rangle .
$$

4. (Estimator for the number of joint transitions.) $\operatorname{Set} \mathcal{L}_{0}^{k, j i}:=0$ and, for $t \geq 1$, define $\mathcal{L}_{t}^{k, j i}$ according to (2.3). We have $\widehat{\mathcal{L}^{k, j i} X_{0}}=0$ and, for any $t \geq 0$,

$$
\begin{aligned}
{\widehat{\mathcal{L}^{k, j i}}}_{t+1}= & \sum_{l=1}^{m}\left(\frac{\boldsymbol{S}\left(t ; \widehat{\mathcal{L}^{k, j i} X_{t}}, Y_{t}\right) \boldsymbol{f}_{l}}{\boldsymbol{1}^{\top} \boldsymbol{S}\left(t ; \hat{X}_{t}, Y_{t}\right) \boldsymbol{f}_{l}}\right)^{+}\left\langle Y_{t+1}, \boldsymbol{f}_{l}\right\rangle \\
& +\left(\frac{\boldsymbol{e}_{j}^{\top} \boldsymbol{S}\left(t ; \boldsymbol{e}_{i}, Y_{t}\right) \boldsymbol{f}_{k}}{\mathbf{1}^{\top} \boldsymbol{S}\left(t ; \hat{X}_{t}, Y_{t}\right) \boldsymbol{f}_{k}}\left\langle\hat{X}_{t}, \boldsymbol{e}_{i}\right\rangle \boldsymbol{e}_{j}\right)^{+}\left\langle Y_{t+1}, \boldsymbol{f}_{k}\right\rangle .
\end{aligned}
$$

The equalities (2.10)-(2.13) are equalities up to a set of P-probability 0. 


\section{Hidden Markov chains}

Let us consider a bivariate Markov chain $(X, Y):=\left(X_{t}, Y_{t}\right)_{t \geq 0} . X_{0}$ and $Y_{0}$ are assumed to be independent random variables. Suppose that the transition probabilities of $(X, Y)$ satisfy

$$
\begin{array}{r}
\mathrm{P}\left(\left(X_{t+1}, Y_{t+1}\right)=\left(\boldsymbol{e}_{j}, \boldsymbol{f}_{k}\right) \mid X_{t}, Y_{t}\right)=\mathrm{P}\left(\left(X_{t+1}, Y_{t+1}\right)=\left(\boldsymbol{e}_{j}, \boldsymbol{f}_{k}\right) \mid X_{t}\right) \\
\Leftrightarrow \mathrm{E}\left[\left\langle X_{t+1}, \boldsymbol{e}_{j}\right\rangle\left\langle Y_{t+1}, \boldsymbol{f}_{k}\right\rangle \mid X_{t}, Y_{t}\right]=\mathrm{E}\left[\left\langle X_{t+1}, \boldsymbol{e}_{j}\right\rangle\left\langle Y_{t+1}, \boldsymbol{f}_{k}\right\rangle \mid X_{t}\right] \\
t \geq 0, k=1, \ldots, m, j=1, \ldots, n .
\end{array}
$$

For any $t \geq 0$, let us define the matrices $\boldsymbol{D}_{k}(t), k=1, \ldots, m$, via their components $D_{k}(t ; \cdot, \cdot)$ by

$$
\mathrm{P}\left(\left(X_{t+1}, Y_{t+1}\right)=\left(\boldsymbol{e}_{j}, \boldsymbol{f}_{k}\right) \mid X_{t}=\boldsymbol{e}_{i}\right)=D_{k}(t ; j, i), \quad i, j=1, \ldots, n .
$$

It is immediately clear that $X$ is a Markov chain with transition matrices $(\boldsymbol{P}(t))_{t \geq 0}$ given by

$$
\boldsymbol{P}(t):=\sum_{k=1}^{m} \boldsymbol{D}_{k}(t) .
$$

For such a Markov chain $(X, Y)$, the random matrix $S\left(t ; X_{t}, Y_{t}\right)$ defined by (2.5) does not depend on $Y_{t}$, and (2.6) becomes

$$
\mathrm{E}\left[X_{t+1} Y_{t+1}^{\top} \mid \mathbb{F}_{t}^{X, Y}\right]=\mathrm{E}\left[X_{t+1} Y_{t+1}^{\top} \mid X_{t}\right]=\boldsymbol{S}\left(t ; X_{t}\right), \quad t \geq 0,
$$

where $S\left(t ; X_{t}\right)$ is the following $n \times m$ random matrix:

$$
\boldsymbol{S}\left(t ; X_{t}\right):=\left(\boldsymbol{D}_{1}(t) X_{t} \cdots \boldsymbol{D}_{m}(t) X_{t}\right), \quad t \geq 0,
$$

i.e. the $j$ th column of $\boldsymbol{S}\left(t ; X_{t}\right)$ is given by $\boldsymbol{D}_{j}(t) X_{t}$. Therefore, the conditional expectation in (2.7) can be written as

$$
\mathrm{E}\left[Y_{t+1} \mid \mathbb{F}_{t}^{X, Y}\right]=\mathrm{E}\left[Y_{t+1} \mid X_{t}\right]=\boldsymbol{S}\left(t ; X_{t}\right)^{\top} \mathbf{1}=G(t) X_{t} \quad \text { for every } t \geq 0,
$$

where $G(t)$ is the $m \times n$ matrix

$$
\boldsymbol{G}(t):=\left(\begin{array}{cc}
\mathbf{1}^{\top} & \boldsymbol{D}_{1}(t) \\
& \vdots \\
\mathbf{1}^{\top} & \boldsymbol{D}_{m}(t)
\end{array}\right)
$$

Since $\mathrm{E}\left[X_{t+1} \mid X_{t}\right]=\boldsymbol{P}(t) X_{t}$, the Markov chain $X$ has the representation

$$
X_{t+1}=\boldsymbol{P}(t) X_{t}+V_{t+1}, \quad t \geq 0,
$$

where $V:=\left(V_{t}\right)_{t \geq 1}$ is an $\mathbb{F}^{X}$-martingale difference (i.e. $V_{t+1}$ is an integrable $\mathbb{F}_{t+1}^{X}$-measurable random variable satisfying $\mathrm{E}\left[V_{t+1} \mid \mathbb{F}_{t}^{X}\right]=\mathbf{0}$ ). Finally, from (3.5) and (3.4), the processes $X$ and $Y$ have the following representation for $t \geq 0$, where $V$ is an $\mathbb{F}^{X}$-martingale difference and $W:=\left(W_{t}\right)_{t \geq 1}$ is an $\mathbb{F}^{X, Y}$-martingale difference:

$$
\begin{aligned}
X_{t+1} & =\boldsymbol{P}(t) X_{t}+V_{t+1}, \\
Y_{t+1} & =\boldsymbol{G}(t) X_{t}+W_{t+1} .
\end{aligned}
$$


The processes $V$ and $W$ are sometimes called the state noise and the observation noise, respectively. In the set-up common in hidden Markov modeling, the noise processes $V$ and $W$ are assumed to be uncorrelated given $X$, that is

$$
\mathrm{E}\left[V_{t+1} W_{t+1}^{\top} \mid X_{t}\right]=\mathbf{0} .
$$

Using (3.2) and the representations (3.6), this last condition may be rewritten as

$$
\begin{aligned}
& \mathrm{E}\left[X_{t+1} Y_{t+1}^{\top} \mid X_{t}\right]=\mathrm{E}\left[X_{t+1} \mid X_{t}\right] \mathrm{E}\left[Y_{t+1} \mid X_{t}\right]^{\top} \\
& \quad \Leftrightarrow \boldsymbol{S}\left(t ; X_{t}\right)=\boldsymbol{P}(t) X_{t}\left(\boldsymbol{G}(t) X_{t}\right)^{\top}=\boldsymbol{P}(t) \operatorname{diag}\left(X_{t}\right) \boldsymbol{G}(t)^{\top} .
\end{aligned}
$$

We refer the reader to [8] for results on filtering in such a context. In this section, we deal with hidden Markov models that only satisfy (3.1). Such processes are referred to as dependent hidden Markov chains. Filtering for time-homogeneous dependent hidden Markov chains was considered in [9, Section 2.10] and [7]. The following result is obtained as a corollary of Theorem 2.1. As mentioned above, the matrix $S\left(t ; X_{t}, Y_{t}\right)$ in (2.7) does not depend on $Y_{t}$ and is replaced by the matrix $\boldsymbol{S}\left(t ; X_{t}\right)$ defined by (3.3). Then the random matrix $\boldsymbol{S}\left(t ; \hat{X}_{t}\right)$ is $\sum_{i=1}^{n} \boldsymbol{S}\left(t ; \boldsymbol{e}_{i}\right)\left\langle\hat{X}_{t}, \boldsymbol{e}_{i}\right\rangle$, according to (2.8), and

$$
\begin{aligned}
\mathbf{1}^{\top} \boldsymbol{S}\left(t ; \hat{X}_{t}\right) \boldsymbol{f}_{k} & =\sum_{i=1}^{n} \mathbf{1}^{\top} \boldsymbol{S}\left(t ; \boldsymbol{e}_{i}\right) \boldsymbol{f}_{k}\left\langle\hat{X}_{t}, \boldsymbol{e}_{i}\right\rangle \\
& =\sum_{i=1}^{n} \mathbf{1}^{\top} \boldsymbol{D}_{k}(t) \boldsymbol{e}_{i}\left\langle\hat{X}_{t}, \boldsymbol{e}_{i}\right\rangle \text { from (3.3) } \\
& =\mathbf{1}^{\top} \boldsymbol{D}_{k}(t) \hat{X}_{t} .
\end{aligned}
$$

Corollary 3.1. 1. (Estimator for the state.) Let $\boldsymbol{x}_{0}$ be a stochastic vector corresponding to the probability distribution of the random variable $X_{0}$. We have

$$
\hat{X}_{0}=\boldsymbol{x}_{0}, \quad \hat{X}_{t+1}=\sum_{k=1}^{m}\left(\frac{\boldsymbol{D}_{k}(t) \hat{X}_{t}}{\mathbf{1}^{\top} \boldsymbol{D}_{k}(t) \hat{X}_{t}}\right)^{+}\left\langle Y_{t+1}, \boldsymbol{f}_{k}\right\rangle, \quad t \geq 0 .
$$

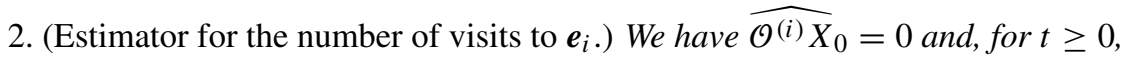

$$
{\widehat{\mathcal{O}^{(i)}}}_{t+1}=\sum_{k=1}^{m}\left(\frac{\boldsymbol{D}_{k}(t) \widehat{\mathcal{O}}(i)_{t}+\left\langle\hat{X}, \boldsymbol{e}_{i}\right\rangle \boldsymbol{D}_{k}(t) \boldsymbol{e}_{i}}{\mathbf{1}^{\top} \boldsymbol{D}_{k}(t) \hat{X}_{t}}\right)^{+}\left\langle Y_{t+1}, \boldsymbol{f}_{k}\right\rangle .
$$

3. (Estimator for the number of jumps of $X$ from $\boldsymbol{e}_{i}$ to $\boldsymbol{e}_{j}$.) We have ${\widehat{N^{j i} X}}_{0}=0$ and, for $t \geq 0$,

$$
{\widehat{N^{j i} X}}_{t+1}=\sum_{k=1}^{m}\left(\frac{\boldsymbol{D}_{k}(t){\widehat{N^{j i} X}}_{t}+D_{k}(t ; j, i)\left\langle\hat{X}_{t}, \boldsymbol{e}_{i}\right\rangle \boldsymbol{e}_{j}}{\boldsymbol{1}^{\top} \boldsymbol{D}_{k}(t) \hat{X}_{t}}\right)^{+}\left\langle Y_{t+1}, \boldsymbol{f}_{k}\right\rangle .
$$

4. (Estimator for the number of joint transitions.) We have $\widehat{\mathcal{L}^{k, j i} X_{0}}=0$ and, for $t \geq 0$,

$$
\begin{aligned}
{\widehat{\mathcal{L}^{k, j i}}}_{t+1}= & \sum_{l=1}^{m}\left(\frac{\boldsymbol{D}_{l}(t) \widehat{\mathcal{L}^{k, j i} X_{t}}}{\mathbf{1}^{\top} \boldsymbol{D}_{l}(t) \hat{X}_{t}}\right)^{+}\left\langle Y_{t+1}, \boldsymbol{f}_{l}\right\rangle \\
& +\left(\frac{D_{k}(t ; j, i)}{\mathbf{1}^{\top} \boldsymbol{D}_{k}(t) \hat{X}_{t}}\left\langle\hat{X}_{t}, \boldsymbol{e}_{i}\right\rangle \boldsymbol{e}_{j}\right)^{+}\left\langle Y_{t+1}, \boldsymbol{f}_{k}\right\rangle .
\end{aligned}
$$


Remark 3.1. 1. A similar estimator for the state was derived by Aström [3] using Bayes's formula.

2. We have obtained a renormalized form of the recursive formulae provided in [7] and [9, Section 2.10] for time-homogeneous hidden Markov chains. It is such a form that is numerically implemented in practice. The simple setting of discrete-time processes with finite state spaces allows us to provide a direct approach. However, the change-of-measure approach used in [7] and [9] is general enough to deal with much more complex hidden Markov models than the ones considered here. Note that, for discrete- or continuous-time hidden Markov chains, the measure change must preserve the Markov property of $X$.

Remark 3.2. The recursion (3.7) may be rewritten as

$$
\hat{X}_{0}=\boldsymbol{x}_{0}, \quad \hat{X}_{t+1}=\boldsymbol{P}(t) \hat{X}_{t}+K_{t}\left(Y_{t+1}-\boldsymbol{G}(t) \hat{X}_{t}\right), \quad t \geq 0,
$$

where $K_{t}$ is the $\mathbb{F}_{t}^{Y}$-measurable matrix-valued random variable defined by

$$
K_{t}:=\left(\left(\frac{\boldsymbol{D}_{1}(t) \hat{X}_{t}}{\mathbf{1}^{\top} \boldsymbol{D}_{1}(t) \hat{X}_{t}}\right)^{+} \cdots\left(\frac{\boldsymbol{D}_{m}(t) \hat{X}_{t}}{\mathbf{1}^{\top} \boldsymbol{D}_{m}(t) \hat{X}_{t}}\right)^{+}\right) .
$$

Indeed, (3.7) has the form

$$
\hat{X}_{t+1}=K_{t} Y_{t+1}
$$

whence

$$
\hat{X}_{t+1}=K_{t}\left(Y_{t+1}-\boldsymbol{G}(t) \hat{X}_{t}\right)+K_{t} \boldsymbol{G}(t) \hat{X}_{t} .
$$

Since

and (see (3.4))

$$
K_{t}=\left(\boldsymbol{D}_{1}(t) \hat{X}_{t} \cdots \boldsymbol{D}_{m}(t) \hat{X}_{t}\right) \operatorname{diag}\left(\left(\frac{1}{\mathbf{1}^{\top} \boldsymbol{D}_{k}(t) \hat{X}_{t}}\right)^{+}\right)
$$

$$
\boldsymbol{G}(t) \hat{X}_{t}=\left(\begin{array}{ccc}
\mathbf{1}^{\top} & \boldsymbol{D}_{1}(t) \hat{X}_{t} \\
\vdots \\
\mathbf{1}^{\top} & \boldsymbol{D}_{m}(t) \hat{X}_{t}
\end{array}\right),
$$

we find that

$$
\begin{aligned}
K_{t} \boldsymbol{G}(t) \hat{X}_{t} & =\left(\boldsymbol{D}_{1}(t) \hat{X}_{t} \cdots \boldsymbol{D}_{m}(t) \hat{X}_{t}\right)\left(\left(\frac{\mathbf{1}^{\top} \boldsymbol{D}_{k}(t) \hat{X}_{t}}{\mathbf{1}^{\top} \boldsymbol{D}_{k}(t) \hat{X}_{t}}\right)^{+}\right)_{k=1}^{m} \\
& =\sum_{k=1}^{m} \boldsymbol{D}_{k}(t) \hat{X}_{t}\left(\frac{\mathbf{1}^{\top} \boldsymbol{D}_{k}(t) \hat{X}_{t}}{\mathbf{1}^{\top} \boldsymbol{D}_{k}(t) \hat{X}_{t}}\right)^{+} .
\end{aligned}
$$

Note that if $\mathbf{1}^{\top} \boldsymbol{D}_{k}(t) \hat{X}_{t}=0$ then $\boldsymbol{D}_{k}(t) \hat{X}_{t}=\mathbf{0}$, meaning that

$$
K_{t} \boldsymbol{G}(t) \hat{X}_{t}=\sum_{k=1}^{m} \boldsymbol{D}_{k}(t) \hat{X}_{t} .
$$

Since $\boldsymbol{P}(t)=\sum_{k=1}^{m} \boldsymbol{D}_{k}(t)$, the proof of (3.8) is complete.

Conditioning with respect to $\mathbb{F}_{t}^{Y}$ in (3.4), we obtain $\boldsymbol{G}(t) \hat{X}_{t}=\mathrm{E}\left[Y_{t+1} \mid \mathbb{F}_{t}^{Y}\right]$. Hence, the random process $\left(K_{t}\right)_{t}$ is the gain with respect to the innovation martingale $\left(Y_{t}-\mathrm{E}\left[Y_{t} \mid \mathbb{F}_{t-1}^{Y}\right]\right)_{t \geq 1}$. Equation (3.8) is the Doob decomposition of the process $\hat{X}$ and can be thought of as the 'projection of the decomposition (3.5) for $X$ onto the internal filtration $\mathbb{F}^{Y}$ '. A related reference is [18]. For each filter in Corollary 3.1, an analogue of (3.8) may be derived in a similar way. 


\section{Parameter estimation for D-BMAPs}

We now consider the discrete-time counterpart of the batch Markovian arrival process (DBMAP) defined by Neuts [14]. For the purposes of this section, only time-homogeneous Markov chains are considered. A standard presentation of a D-BMAP is as follows. Let us consider a Markov chain $(X, N)=\left(X_{t}, N_{t}\right)_{t \geq 0}$ over the state space $E \times \mathbb{N}$, with transition probabilities satisfying

$$
\begin{aligned}
& \mathrm{P}\left(\left(X_{t+1}, N_{t+1}\right)=\left(\boldsymbol{e}_{j}, l+k\right) \mid\left(X_{t}, N_{t}\right)=\left(\boldsymbol{e}_{i}, l\right)\right) \\
& \quad=\mathrm{P}\left(\left(X_{t+1}, N_{t+1}-N_{t}\right)=\left(\boldsymbol{e}_{j}, k\right) \mid X_{t}=\boldsymbol{e}_{i}\right) \\
& \quad=D_{k}(j, i)
\end{aligned}
$$

for all $l \geq 0, t \geq 0, i, j=1, \ldots, n$, and $k=0, \ldots, m$. The other transition probabilities are $0, N_{0}=0$, and the probability distribution of $X_{0}$ is denoted by $\boldsymbol{x}_{0}$. The above matrices $\boldsymbol{D}_{k}, k=0, \ldots, m$, agree with those of Lucantoni's formalism for defining the finite BMAP [2]. The nonnegative entry $D_{0}(j, i)$ represents the probability that $X$ jumps from state $\boldsymbol{e}_{i}$ to $\boldsymbol{e}_{j}$ with no arrival event. For $k \geq 1$, the entry $D_{k}(j, i)$ is the probability that $X$ jumps from state $\boldsymbol{e}_{i}$ to state $\boldsymbol{e}_{j}$ with the occurrence of $k$ arrivals. Then $X$ is a Markov chain with transition probability matrix $\boldsymbol{P}=\sum_{k=0}^{m} \boldsymbol{D}_{k}$. D-BMAPs have been found useful in numerous situations (e.g. see [4] and [1]). We point out the central role played by the $\boldsymbol{D}_{k}$ in analyzing the number $N_{t}$ of arrivals, up to time $t$, generated by a D-BMAP. For instance, the distribution function of $N_{t}$ may be numerically evaluated using the following system of difference equations, where $\boldsymbol{x}(l, t):=\left(\mathrm{P}\left(N_{t} \leq l \mid X_{0}=\boldsymbol{e}_{i}\right)\right)_{i=1}^{n}:$

$$
\boldsymbol{x}(l, t)=\sum_{k=0}^{l \wedge m} \boldsymbol{D}_{k} \boldsymbol{x}(l-k, t-1), \quad l \geq 0, t \geq 1, \quad \boldsymbol{x}(l, 0)=\mathbf{1}, \quad l \geq 0 .
$$

Let $\boldsymbol{\theta}$ be the nonnegative parameter vector $\boldsymbol{\theta}:=\left\{D_{k}(j, i), k=0, \ldots, m, i, j=1, \ldots, n\right\}$ whose entries satisfy

$$
\sum_{k=0}^{m} \sum_{j=1}^{n} D_{k}(j, i)=1 \quad \text { for } i=1, \ldots, n .
$$

The vector $\boldsymbol{\theta}$ must be estimated from the data. We stress that a D-BMAP $(X, N)$ can be thought of as a time-homogeneous hidden Markov chain $(X, Y)$, as defined in Section 3, for which $\mathrm{P}\left(Y_{0}=\boldsymbol{f}_{0}\right)=1$ and

$$
\mathrm{P}\left(\left(X_{t+1}, Y_{t+1}\right)=\left(\boldsymbol{e}_{j}, \boldsymbol{f}_{k}\right) \mid X_{t}=\boldsymbol{e}_{i}\right)=D_{k}(j, i), \quad k=0, \ldots, m, i, j=1, \ldots, n .
$$

Thus, $\boldsymbol{f}_{k}$ stands for a $k$-arrival event $(k=0, \ldots, m)$. In what follows, a D-BMAP is identified with the associated hidden Markov chain $(X, Y)$ defined above. The EM-algorithm is known to be a standard procedure for the statistical estimation of discrete-time hidden Markov chains [15].

Methods of statistical estimation for the Markovian arrival process have been recently developed in the continuous-time context. Specifically, the EM-algorithm has been used by Rydén [16] for the Markov-modulated Poisson process, by Asmussen [2] for phase-type distributions (see also the references of [2]), by Klemm et al. [12] for general Markovian arrival processes, and by Breuer [6] for BMAPs. The numerical experiments reported in these studies show that the EM-algorithm works well, in general. In these works, the number of states of $X$ is assumed to be known. Procedures for estimating the number $n$ of states of $X$ were discussed in [17] (a brief discussion on the order estimation was also presented in [6, p. 124]). 
We briefly explain the EM-algorithm for D-BMAPs. For a fixed parameter vector $\boldsymbol{\theta}$, the underlying probability measure and the associated expectation are denoted by $\mathrm{P}_{\boldsymbol{\theta}}$ and $\mathrm{E}_{\boldsymbol{\theta}}$, respectively. The random variable $X_{0}$, or its probability distribution $\boldsymbol{x}_{0}$, is assumed to be known. Under $\mathrm{P}_{\boldsymbol{\theta}}$, the likelihood function for the complete data $(X, Y)$, up to time $t$, is

$$
L_{t}(\boldsymbol{\theta} ; X, Y):=\prod_{l=1}^{t} \prod_{k=0}^{m} \prod_{i, j=1}^{n} D_{k}(j, i)^{\left\langle Y_{l}, \boldsymbol{f}_{k}\right\rangle\left\langle X_{l}, \boldsymbol{e}_{j}\right\rangle\left\langle X_{l-1}, \boldsymbol{e}_{i}\right\rangle} \prod_{i=1}^{n} x_{0}(i)^{\left\langle X_{0}, \boldsymbol{e}_{i}\right\rangle},
$$

The parameter $\boldsymbol{\theta}$ is estimated from the observations $\left(Y_{1}, \ldots, Y_{t}\right)$, using the following iterative procedure, where the vector $\boldsymbol{\theta}_{p}:=\left\{D_{k}^{(p)}(i, j), i, j=1, \ldots, n, k=0, \ldots, m\right\}$ denotes the estimate of $\boldsymbol{\theta}$ after the $p$ th iteration.

1. Initialization. Choose $\boldsymbol{\theta}_{0}$.

2. Expectation step. Set $\boldsymbol{\theta}=\boldsymbol{\theta}_{p}$. Consider the so-called 'pseudo-log-likelihood function' $Q\left(\boldsymbol{\theta}^{*} \mid \boldsymbol{\theta}\right)$, defined by

$$
Q\left(\boldsymbol{\theta}^{*} \mid \boldsymbol{\theta}\right):=\mathrm{E}_{\boldsymbol{\theta}}\left[\log L_{t}\left(\boldsymbol{\theta}^{*} ; X, Y\right) \mid \mathbb{F}_{t}^{Y}\right]=\sum_{k=0}^{m} \sum_{i, j=1}^{n} \log D_{k}^{*}(j, i) \widehat{\mathcal{L}^{k, j i}}{ }_{t}+K,
$$

where

$$
\boldsymbol{\theta}^{*}:=\left\{D_{k}^{*}(j, i), k=0, \ldots, m, i, j=1, \ldots, n\right\},
$$

$K$ does not depend on $\boldsymbol{\theta}^{*}$, and $\widehat{\mathcal{L}^{k, j i}}{ }_{t}:=\mathrm{E}_{\boldsymbol{\theta}}\left[\mathcal{L}_{t}^{k, j i} \mid \mathbb{F}_{t}^{Y}\right]$.

3. Maximization step. Determine the $\boldsymbol{\theta}_{p+1}$ that maximizes the function (4.1) under the constraints

$$
\sum_{i=1}^{n} \widehat{\mathcal{O}^{(i)}} t \sum_{k=0}^{m} \sum_{j=1}^{n} D_{k}^{*}(j, i)=t
$$

where $\widehat{\mathcal{O}^{(i)}} t=\mathrm{E}_{\boldsymbol{\theta}}\left[\mathcal{O}_{t}^{(i)} \mid \mathbb{F}_{t}^{Y}\right]$. We obtain

$$
D_{k}^{(p+1)}(j, i)=\frac{\widehat{\mathcal{L}^{k, j i}} t}{\widehat{\mathcal{O}^{(i)} t}}, \quad i, j=1, \ldots, n .
$$

4. Return to step 2 until a stopping criterion is satisfied.

It is clear that the best estimate of the transition probability $P(j, i)$ after the $(p+1)$ th iteration is given by

$$
P^{(p+1)}(j, i)=\frac{\widehat{N^{j i}} t}{\widehat{\mathcal{O}^{(i)}} t}, \quad i, j=1, \ldots, n .
$$

The EM-algorithm produces a sequence of parameter vectors $\boldsymbol{\theta}_{0}, \boldsymbol{\theta}_{1}, \ldots$ such that [10]

- the sequence of values of the observed likelihood function $\left(l_{t}\left(\boldsymbol{\theta}_{p} ; Y\right)\right)_{p}$ is nondecreasing (with equality if and only if $\boldsymbol{\theta}_{p+1}=\boldsymbol{\theta}_{p}$ under an identifiability condition), and

- under appropriate conditions, the sequence $\left(l_{t}\left(\boldsymbol{\theta}_{p} ; Y\right)\right)_{p}$ converges to a local maximum of the observed likelihood function.

Note that the zero entries of the $\boldsymbol{D}_{k}$ are preserved by the procedure above. 
As a result of the procedure above, we must compute the estimates in (4.2). The standard method is to use the Baum-Welch implementation of the maximization step of the EM-algorithm (also referred to as the 'forward-backward' technique). This was done in the previously mentioned works on continuous-time BMAPs [2], [6], [12]. In the filter-based approach pioneered by Elliott [7], [9], the estimates (4.2) are directly computed from the recursive equations given in Corollary 3.1. Therefore, the filter-based EM-procedure is very easily implemented and retains the well-established statistical properties of the EM-algorithm. The basic difference from the standard Baum-Welch method is that only one pass through the dataset is needed in the filter-based method. This approach was shown to be of value for specific continuous-time Markovian models in a recent paper [13].

We mention that a simple procedure for estimating the matrices $\boldsymbol{D}_{k}$ associated with a continuous-time BMAP was numerically compared to the EM-algorithm in [6]. Breuer's procedure [6] was shown to be much faster and to require less storage space than the BaumWelch implementation of the EM-algorithm. Breuer provided no details on the computational complexity or the statistical properties of his procedure. A discrete-time version of this simple procedure could be designed. We mention that the storage space required by the filter-based implementation of the EM-algorithm is independent of the number of observations (indeed, only the filtered variables at each instant need be stored in order to compute the variables at the next instant). The filter-based EM-algorithm has a slightly higher computational complexity than its Baum-Welch form. Therefore, Breuer's procedure should perform faster than ours. However, due to the simplicity of the filter-based EM-algorithm for D-BMAPs, the speed increase should not be very great. We will not go into further details of Breuer's procedure.

\section{Proof of Theorem 2.1}

A formula for the conditional expectation given $\mathbb{F}_{t}^{Y}, t \geq 0$, is proposed in the lemma below. Equation (5.5) is our basic formula for deriving the recursive equations reported in Theorem 2.1. It is based on the natural dynamics of the observations:

$$
\mathbb{F}_{t+1}^{Y}=\mathbb{F}_{t}^{Y} \vee \sigma\left(Y_{t+1}\right)
$$

Here, $\sigma\left(Y_{t+1}\right)$ is a $\sigma$-subalgebra of $\mathcal{F}$ generated by the finite partition $\left\{A_{1}, \ldots, A_{m}\right\}$ of $\Omega$, with

$$
A_{k}:=\left\{Y_{t+1}=\boldsymbol{f}_{k}\right\}
$$

The conditional probability given $A_{k}$ is defined, for any $B \in \mathcal{F}$, by

$$
\mathrm{P}_{A_{k}}(B):=\left(\frac{1}{\mathrm{P}\left(A_{k}\right)}\right)^{+} \mathrm{E}\left[1_{B \cap A_{k}}\right]
$$

Then, for any integrable random variable $X$, we have

$$
\begin{aligned}
\mathrm{E}_{A_{k}}[X] & =\left(\frac{1}{\mathrm{P}\left(A_{k}\right)}\right)^{+} \mathrm{E}\left[X 1_{A_{k}}\right], \\
\mathrm{E}\left[X \mid \sigma\left(Y_{t+1}\right)\right] & =\sum_{k=1}^{m} \mathrm{E}_{A_{k}}[X] 1_{A_{k}} .
\end{aligned}
$$


Lemma 5.1. Consider the filtration $\mathbb{F}^{Y}=\left(\mathbb{F}_{t}^{Y}\right)_{t \geq 0}$ of $\mathcal{F}$ and an integrable random variable $X$. The following relations hold for every $t \geq 0$ :

$$
\begin{aligned}
\mathrm{E}\left[X \mid \mathbb{F}_{t}^{Y} \vee \sigma\left(Y_{t+1}\right)\right] & =\sum_{k=1}^{m} \mathrm{E}_{A_{k}}\left[X \mid \mathbb{F}_{t}^{Y}\right] 1_{A_{k}}, \\
\mathrm{E}\left[1_{A_{k}} X \mid \mathbb{F}_{t}^{Y}\right] & =\mathrm{E}\left[1_{A_{k}} \mid \mathbb{F}_{t}^{Y}\right] \mathrm{E}_{A_{k}}\left[X \mid \mathbb{F}_{t}^{Y}\right], \\
\mathrm{E}\left[X \mid \mathbb{F}_{t}^{Y} \vee \sigma\left(Y_{t+1}\right)\right] & =\sum_{k=1}^{m}\left(\frac{\mathrm{E}\left[1_{A_{k}} X \mid \mathbb{F}_{t}^{Y}\right]}{\mathrm{E}\left[1_{A_{k}} \mid \mathbb{F}_{t}^{Y}\right]}\right)^{+} 1_{A_{k}} .
\end{aligned}
$$

Remark 5.1. Lemma 5.1 still holds when the random variable $Y_{t+1}$ is replaced by any finitevalued random variable. A general form of (5.4) is referred to as a conditional Bayes's formula in [9].

Proof of Lemma 5.1. Let $t$ be a positive integer. Any element of the $\sigma$-algebra $\mathbb{F}_{t}^{Y} \vee \sigma\left(Y_{t+1}\right)$ is a finite union of sets of the form $B \cap A_{k}$ for some $B \in \mathbb{F}_{t}^{Y}$. The summation on the right-hand side of (5.3) is $\left(\mathbb{F}_{t}^{Y} \vee \sigma\left(Y_{t+1}\right)\right)$-measurable. Hence, to derive (5.3), we must show that, for $k=1, \ldots, m$,

$$
\mathrm{E}\left[1_{B \cap A_{k}} X\right]=\mathrm{E}\left[1_{B \cap A_{k}} \sum_{i=1}^{m} 1_{A_{i}} \mathrm{E}_{A_{i}}\left[X \mid \mathbb{F}_{t}^{Y}\right]\right], \quad B \in \mathbb{F}_{t}^{Y} .
$$

We can write

$$
\begin{aligned}
\mathrm{E} & {\left[1_{B \cap A_{k}} X\right] } \\
& =\mathrm{P}\left(A_{k}\right) \mathrm{E}_{A_{k}}\left[1_{B} X\right] \quad(\text { from }(5.1)) \\
& =\mathrm{P}\left(A_{k}\right) \mathrm{E}_{A_{k}}\left[\mathrm{E}_{A_{k}}\left[1_{B} X \mid \mathbb{F}_{t}^{Y}\right]\right] \\
& =\mathrm{P}\left(A_{k}\right) \mathrm{E}_{A_{k}}\left[1_{B} \mathrm{E}_{A_{k}}\left[X \mid \mathbb{F}_{t}^{Y}\right]\right] \quad\left(\text { since } B \in \mathbb{F}_{t}^{Y}\right) \\
& =\mathrm{E}\left[1_{B} \mathrm{E}_{A_{k}}\left[X \mid \mathbb{F}_{t}^{Y}\right] 1_{A_{k}}\right] \quad\left(\text { by applying }(5.1) \text { to the random variable } 1_{B} \mathrm{E}_{A_{k}}\left[X \mid \mathbb{F}_{t}^{Y}\right]\right) \\
& =\mathrm{E}\left[1_{B \cap A_{k}} \sum_{i=1}^{m} 1_{A_{i}} \mathrm{E}_{A_{i}}\left[X \mid \mathbb{F}_{t}^{Y}\right]\right] \quad\left(\text { since } 1_{A_{k} \cap A_{i}} \text { vanishes except when } k=i\right) .
\end{aligned}
$$

Second, we deduce from

$$
\mathrm{E}\left[X \mid \mathbb{F}_{t}^{Y}\right]=\mathrm{E}\left[\mathrm{E}\left[X \mid \mathbb{F}_{t}^{Y} \vee \sigma\left(Y_{t+1}\right)\right] \mid \mathbb{F}_{t}^{Y}\right]
$$

and (5.3) that

$$
\mathrm{E}\left[X \mid \mathbb{F}_{t}^{Y}\right]=\sum_{i=1}^{m} \mathrm{E}\left[1_{A_{i}} \mid \mathbb{F}_{t}^{Y}\right] \mathrm{E}_{A_{i}}\left[X \mid \mathbb{F}_{t}^{Y}\right] .
$$

Applying this equality to the random variable $1_{A_{k}} X$, we obtain (5.4).

Note that if $\mathrm{P}\left(A_{k}\right)=0$ for some $k$, then $\mathrm{E}_{A_{k}}\left[X \mid \mathbb{F}_{t}^{Y}\right]=0$. Hence, in (5.3), each term $1_{A_{k}} \mathrm{E}_{A_{k}}\left[X \mid \mathbb{F}_{t}^{Y}\right]$ for which $\mathrm{P}\left(A_{k}\right)=0$ vanishes. We also mention that $\mathrm{P}\left(A_{k}\right)>0$ implies that $\mathrm{P}\left(A_{k} \mid \mathbb{F}_{t}^{Y}\right)=\mathrm{E}\left[1_{A_{k}} \mid \mathbb{F}_{t}^{Y}\right]>0, \mathrm{P}$ almost surely Therefore, by (5.4), a nonzero term in (5.3) may be written as the following well-defined fraction (up to a set of P-probability 0 ):

$$
\frac{\mathrm{E}\left[1_{A_{k}} X \mid \mathbb{F}_{t}^{Y}\right]}{\mathrm{E}\left[1_{A_{k}} \mid \mathbb{F}_{t}^{Y}\right]}
$$


Formula (5.5) is just a reformulation of (5.3) taking into account (5.4) and the previous comments. This completes the proof.

Proof of Theorem 2.1. For $t=0$, we deduce from (5.1) and (5.2) that

$$
\begin{aligned}
\hat{X}_{0}:=\mathrm{E}\left[X_{0} \mid Y_{0}\right] & =\sum_{k=1}^{m}\left(\frac{\mathrm{E}\left[X_{0}\left\langle Y_{0}, \boldsymbol{f}_{k}\right\rangle\right]}{\mathrm{E}\left[\left\langle Y_{0}, \boldsymbol{f}_{k}\right\rangle\right]}\right)^{+}\left\langle Y_{0}, \boldsymbol{f}_{k}\right\rangle \\
& =\sum_{k=1}^{m}\left(\frac{\boldsymbol{p}_{k}}{\mathbf{1}^{\top} \boldsymbol{p}_{k}}\right)^{+}\left\langle Y_{0}, \boldsymbol{f}_{k}\right\rangle,
\end{aligned}
$$

with the notation introduced in Theorem 2.1.

An application of Lemma 5.1 allows us to complete the proof of (2.10). Indeed, we find from our basic formula (5.5) that, for any $t \geq 0$,

$$
\mathrm{E}\left[X_{t+1} \mid \mathbb{F}_{t}^{Y} \vee \sigma\left(Y_{t+1}\right)\right]=\sum_{k=1}^{m}\left(\frac{\mathrm{E}\left[X_{t+1}\left\langle Y_{t+1}, \boldsymbol{f}_{k}\right\rangle \mid \mathbb{F}_{t}^{Y}\right]}{\mathrm{E}\left[\left\langle Y_{t+1}, \boldsymbol{f}_{k}\right\rangle \mid \mathbb{F}_{t}^{Y}\right]}\right)^{+}\left\langle Y_{t+1}, \boldsymbol{f}_{k}\right\rangle
$$

The numerator of the fraction above may be rewritten as

$$
\begin{aligned}
\mathrm{E}\left[X_{t+1}\left\langle Y_{t+1}, \boldsymbol{f}_{k}\right\rangle \mid \mathbb{F}_{t}^{Y}\right] & =\mathrm{E}\left[\mathrm{E}\left[X_{t+1} Y_{t+1}^{\top} \boldsymbol{f}_{k} \mid \mathbb{F}_{t}^{X, Y}\right] \mid \mathbb{F}_{t}^{Y}\right] \\
& =\mathrm{E}\left[\mathrm{E}\left[X_{t+1} Y_{t+1}^{\top} \mid \mathbb{F}_{t}^{X, Y}\right] \mid \mathbb{F}_{t}^{Y}\right] \boldsymbol{f}_{k} \\
& =\mathrm{E}\left[\boldsymbol{S}\left(t ; X_{t}, Y_{t}\right) \mid \mathbb{F}_{t}^{Y}\right] \boldsymbol{f}_{k} \quad(\text { from (2.6)) } \\
& =\boldsymbol{S}\left(t ; \hat{X}_{t}, Y_{t}\right) \boldsymbol{f}_{k} \quad(\operatorname{using}(2.9)) .
\end{aligned}
$$

Since $\mathbf{1}^{\top} X_{t+1}=1$, the scalar $\mathrm{E}\left[\left\langle Y_{t+1}, \boldsymbol{f}_{k}\right\rangle \mid \mathbb{F}_{t}^{Y}\right]$ equals $\mathbf{1}^{\top} \mathrm{E}\left[X_{t+1}\left\langle Y_{t+1}, \boldsymbol{f}_{k}\right\rangle \mid \mathbb{F}_{t}^{Y}\right]$ or, by the previous result, $\mathbf{1}^{\top} \boldsymbol{S}\left(t ; \hat{X}_{t}, Y_{t}\right) \boldsymbol{f}_{k}$. From this, we recover (2.10).

Recall that the scalar $\mathcal{L}_{t}^{k, j i}$ is defined by

$$
\mathcal{L}_{t}^{k, j i}=\sum_{l=1}^{t}\left\langle Y_{l}, \boldsymbol{f}_{k}\right\rangle\left\langle X_{l}, \boldsymbol{e}_{j}\right\rangle\left\langle X_{l-1}, \boldsymbol{e}_{i}\right\rangle
$$

for $t \geq 1$. Thus, we find that

$$
\mathcal{L}_{t+1}^{k, j i}=\mathcal{L}_{t}^{k, j i}+\left\langle Y_{t+1}, \boldsymbol{f}_{k}\right\rangle\left\langle X_{t+1}, \boldsymbol{e}_{j}\right\rangle\left\langle X_{t}, \boldsymbol{e}_{i}\right\rangle
$$

The corresponding decomposition of the vector $\mathcal{L}_{t+1}^{k, j i} X_{t+1}$ therefore allows us to write

$$
\begin{aligned}
\widehat{\mathcal{L}^{k, j i} X_{t+1}} & :=\mathrm{E}\left[\mathcal{L}_{t+1}^{k, j i} X_{t+1} \mid \mathbb{F}_{t+1}^{Y}\right] \\
& =\mathrm{E}\left[\mathcal{L}_{t}^{k, j i} X_{t+1} \mid \mathbb{F}_{t+1}^{Y}\right]+\mathrm{E}\left[\left\langle Y_{t+1}, \boldsymbol{f}_{k}\right\rangle\left\langle X_{t+1}, \boldsymbol{e}_{j}\right\rangle\left\langle X_{t}, \boldsymbol{e}_{i}\right\rangle X_{t+1} \mid \mathbb{F}_{t+1}^{Y}\right] \\
& =\mathrm{E}\left[\mathcal{L}_{t}^{k, j i} X_{t+1} \mid \mathbb{F}_{t+1}^{Y}\right]+\left\langle Y_{t+1}, \boldsymbol{f}_{k}\right\rangle \mathrm{E}\left[\left\langle X_{t+1}, \boldsymbol{e}_{j}\right\rangle\left\langle X_{t}, \boldsymbol{e}_{i}\right\rangle \mid \mathbb{F}_{t+1}^{Y}\right] \boldsymbol{e}_{j} .
\end{aligned}
$$

Using (5.5), the first term on the right-hand side of the above equality has the form

$$
\sum_{l=1}^{m}\left(\frac{\mathrm{E}\left[\mathcal{L}_{t}^{k, j i} X_{t+1}\left\langle Y_{t+1}, \boldsymbol{f}_{l}\right\rangle \mid \mathbb{F}_{t}^{Y}\right]}{\mathrm{E}\left[\left\langle Y_{t+1}, \boldsymbol{f}_{l}\right\rangle \mid \mathbb{F}_{t}^{Y}\right]}\right)^{+}\left\langle Y_{t+1}, \boldsymbol{f}_{l}\right\rangle
$$


Let us develop the numerator of the above fraction. Since $\mathcal{L}_{t}^{k, j i}$ is $\mathbb{F}_{t}^{X, Y}$-measurable, we have

$$
\begin{aligned}
\mathrm{E}\left[\mathcal{L}_{t}^{k, j i}\right. & \left.X_{t+1}\left\langle Y_{t+1}, \boldsymbol{f}_{l}\right\rangle \mid \mathbb{F}_{t}^{Y}\right] \\
= & \mathrm{E}\left[\mathcal{L}_{t}^{k, j i} \mathrm{E}\left[X_{t+1} Y_{t+1}^{\top} \boldsymbol{f}_{l} \mid \mathbb{F}_{t}^{X, Y}\right] \mid \mathbb{F}_{t}^{Y}\right] \\
= & \mathrm{E}\left[\mathcal{L}_{t}^{k, j i} \mathrm{E}\left[X_{t+1} Y_{t+1}^{\top} \mid X_{t}, Y_{t}\right] \mid \mathbb{F}_{t}^{Y}\right] \boldsymbol{f}_{l} \quad \text { (since }(X, Y) \text { is Markovian) } \\
= & \mathrm{E}\left[\mathcal{L}_{t}^{k, j i} \boldsymbol{S}\left(t ; X_{t}, Y_{t}\right) \mid \mathbb{F}_{t}^{Y}\right] \boldsymbol{f}_{l} \quad(\text { from }(2.6)) \\
= & \sum_{p=1}^{n} \mathrm{E}\left[\mathcal{L}_{t}^{k, j i}\left\langle X_{t}, \boldsymbol{e}_{p}\right\rangle \boldsymbol{S}\left(t ; \boldsymbol{e}_{p}, Y_{t}\right) \mid \mathbb{F}_{t}^{Y}\right] \boldsymbol{f}_{l} \\
= & \sum_{p=1}^{n} \mathrm{E}\left[\mathcal{L}_{t}^{k, j i}\left\langle X_{t}, \boldsymbol{e}_{p}\right\rangle \mid \mathbb{F}_{t}^{Y}\right] \boldsymbol{S}\left(t ; \boldsymbol{e}_{p}, Y_{t}\right) \boldsymbol{f}_{l} \\
= & \sum_{p=1}^{n}\left\langle\mathrm{E}\left[\mathcal{L}_{t}^{k, j i} X_{t} \mid \mathbb{F}_{t}^{Y}\right], \boldsymbol{e}_{p}\right\rangle \boldsymbol{S}\left(t ; \boldsymbol{e}_{p}, Y_{t}\right) \boldsymbol{f}_{l} \\
= & \boldsymbol{S}\left(t ; \mathcal{L}^{k, j i} X_{t}, Y_{t}\right) \boldsymbol{f}_{l} \quad(\text { using }(2.8)) .
\end{aligned}
$$

We now deduce, from (5.5), that the scalar $\left\langle Y_{t+1}, \boldsymbol{f}_{k}\right\rangle \mathrm{E}\left[\left\langle X_{t+1}, \boldsymbol{e}_{j}\right\rangle\left\langle X_{t}, \boldsymbol{e}_{i}\right\rangle \mid \mathbb{F}_{t+1}^{Y}\right]$ is equal to

$$
\left(\frac{\mathrm{E}\left[\left\langle X_{t+1}, \boldsymbol{e}_{j}\right\rangle\left\langle X_{t}, \boldsymbol{e}_{i}\right\rangle\left\langle Y_{t+1}, \boldsymbol{f}_{k}\right\rangle \mid \mathbb{F}_{t}^{Y}\right]}{\mathrm{E}\left[\left\langle Y_{t+1}, \boldsymbol{f}_{k}\right\rangle \mid \mathbb{F}_{t}^{Y}\right]}\right)^{+}\left\langle Y_{t+1}, \boldsymbol{f}_{k}\right\rangle .
$$

The scalar in the numerator of the fraction above is rewritten as follows:

$$
\begin{aligned}
\mathrm{E}\left[\left\langle X_{t+1}, \boldsymbol{e}_{j}\right\rangle\left\langle X_{t}, \boldsymbol{e}_{i}\right\rangle\left\langle Y_{t+1}, \boldsymbol{f}_{k}\right\rangle \mid \mathbb{F}_{t}^{Y}\right] & =\mathrm{E}\left[\left\langle X_{t}, \boldsymbol{e}_{i}\right\rangle \mathrm{E}\left[\left\langle X_{t+1}, \boldsymbol{e}_{j}\right\rangle\left\langle Y_{t+1}, \boldsymbol{f}_{k}\right\rangle \mid \mathbb{F}_{t}^{X, Y}\right] \mid \mathbb{F}_{t}^{Y}\right] \\
& =\mathrm{E}\left[\left\langle X_{t}, \boldsymbol{e}_{i}\right\rangle \mathrm{E}\left[e_{j}^{\top} X_{t+1} Y_{t+1}^{\top} \boldsymbol{f}_{k} \mid X_{t}, Y_{t}\right] \mid \mathbb{F}_{t}^{Y}\right] \\
& =\boldsymbol{e}_{j}^{\top} \mathrm{E}\left[\left\langle X_{t}, \boldsymbol{e}_{i}\right\rangle \mathrm{E}\left[X_{t+1} Y_{t+1}^{\top} \mid X_{t}, Y_{t}\right] \mid \mathbb{F}_{t}^{Y}\right] \boldsymbol{f}_{k} \\
& =\boldsymbol{e}_{j}^{\top} \mathrm{E}\left[\left\langle X_{t}, \boldsymbol{e}_{i}\right\rangle \boldsymbol{S}\left(t ; \boldsymbol{e}_{i}, Y_{t}\right) \mid \mathbb{F}_{t}^{Y}\right] \boldsymbol{f}_{k} \quad \text { (from (2.6)) } \\
& =\boldsymbol{e}_{j}^{\top} \boldsymbol{S}\left(t ; \boldsymbol{e}_{i}, Y_{t}\right) \boldsymbol{f}_{k} \mathrm{E}\left[\left\langle X_{t}, \boldsymbol{e}_{i}\right\rangle \mid \mathbb{F}_{t}^{Y}\right] \\
& =\boldsymbol{e}_{j}^{\top} \boldsymbol{S}\left(t ; \boldsymbol{e}_{i}, Y_{t}\right) \boldsymbol{f}_{k}\left\langle\hat{X}_{t}, \boldsymbol{e}_{i}\right\rangle .
\end{aligned}
$$

Since $\mathrm{E}\left[\left\langle Y_{t+1}, \boldsymbol{f}_{k}\right\rangle \mid \mathbb{F}_{t}^{Y}\right]$ was found to be $\mathbf{1}^{\top} \boldsymbol{S}\left(t ; \hat{X}_{t}, Y_{t}\right) \boldsymbol{f}_{k}$, it follows that

$$
\left\langle Y_{t+1}, \boldsymbol{f}_{k}\right\rangle \mathrm{E}\left[\left\langle X_{t+1}, \boldsymbol{e}_{j}\right\rangle\left\langle X_{t}, \boldsymbol{e}_{i}\right\rangle \mid \mathbb{F}_{t+1}^{Y}\right]=\left(\frac{\boldsymbol{e}_{j}^{\top} \boldsymbol{S}\left(t ; \boldsymbol{e}_{i}, Y_{t}\right) \boldsymbol{f}_{k}}{\mathbf{1}^{\top} \boldsymbol{S}\left(t ; \hat{X}_{t}, Y_{t}\right) \boldsymbol{f}_{k}}\left\langle\hat{X}_{t}, \boldsymbol{e}_{i}\right\rangle\right)^{+}\left\langle Y_{t+1}, \boldsymbol{f}_{k}\right\rangle .
$$

Therefore, the recursion (2.13) for $\widehat{\mathcal{L}^{k, j i} X}$ finally takes the form of (5.6), using (5.7) and (5.8).

A direct proof of (2.11) and (2.12) could be given using Lemma 5.1. For the sake of brevity, we remark that they can easily be derived from (2.13), which is associated with the filter $\widehat{\mathcal{L}^{k, j i} X}$, due to the relations

$$
\begin{aligned}
& \widehat{\mathcal{O}}^{(i)} X_{t+1}=\sum_{k=1}^{m} \sum_{j=1}^{n}{\widehat{\mathcal{L}^{k, j i}}}_{t+1}, \\
& {\widehat{N^{j i} X_{t+1}}}=\sum_{k=1}^{m}{\widehat{\mathcal{L}^{k, j i}}}_{t+1} .
\end{aligned}
$$




\section{Conclusion}

In this note, we have derived normalized filters for various statistics of any partially observed discrete-time finite Markov chain in recursive form. These results cover, in particular, the case of time-homogeneous dependent hidden Markov chains discussed in [7] and [9]. We emphasize that only simple computations involving discrete-time Markov chains are required. An application to the parameter estimation for discrete-time batch Markovian arrival processes has been outlined. Models of this sort are widely used in applied probability, especially in queueing theory.

\section{References}

[1] Alfa, A. S. and Neuts, M. (1995). Modelling vehicular traffic using the discrete time Markovian arrival process. Transportation Sci. 29, 109-117.

[2] Asmussen, S. (2000). Matrix-analytic models and their analysis. Scand. J. Statist. 27, 193-226.

[3] Aström, K. J. (1965). Optimal control of Markov processes with incomplete state information. J. Math. Anal. Appl. 10, 174-205.

[4] Blondia, C. (1993). A discrete-time batch Markovian arrival process as B-ISDN traffic model. Belgian J. Operat. Res. Statist. Comput. Sci. 32, 3-23.

[5] Boucheron, S. And Gassiat, E. (2005). An information theoretic perspective on order estimation. In Inference in Hidden Markov Models, eds O. Cappé, E. Moulines and T. Rydén. Springer, New York.

[6] Breuer, L. (2002). An EM algorithm for batch arrival processes and its comparison to a simpler estimation procedure. Ann. Operat. Res. 112, 123-138.

[7] Elliott, R. J. (1995). Recursive estimation for hidden Markov models: a dependent case. Stoch. Anal. Appl. 13, 437-460.

[8] Elliott, R. J. and Huang, H. (1994). How to count and guess well: discrete adaptative filters. Appl. Math. Optimization 30, 51-78.

[9] Elliott, R. J., Aggoun, L. And Moore, J. B. (1995). Hidden Markov Models (Appl. Math. (New York) 29). Springer, New York.

[10] Ephraim, Y. and Merhav, N. (2002). Hidden Markov processes. IEEE Trans. Inf. Theory 48, 1518-1569.

[11] Gassiat, E. AND Boucheron S. (2003). Optimal error exponents for hidden Markov model order estimation. IEEE Trans. Inf. Theory 49, 864-880.

[12] Klemm, A., Lindemann, C. And Lohmann, M. (2003). Modeling IP traffic using the batch Markovian arrival process. Performance Evaluation 54, 149-173.

[13] Lin, D. AND MaKis, V. (2003). Filters for a partially observable system subject to random failure. Adv. Appl. Prob. 35, 207-227.

[14] Neuts, M. F. (1989). Structured Stochastic Matrices of M/G/1 Type and Their Applications. Marcel Dekker, New York.

[15] RABINER, L. R. (1989). A tutorial on hidden Markov models and selected applications in speech recognition. Proc. IEEE 77, 257-286.

[16] RydÉn, T. (1996). An EM algorithm for estimation in Markov-modulated Poisson processes. Comput. Statist. Data Anal. 21, 431-447.

[17] RYDÉN, T. (1997). Estimating the order of continuous phase-type distributions and Markov-modulated Poisson processes. Stoch. Models 13, 417-433.

[18] Segall, A. (1976). Recursive estimation from discrete-time point process. IEEE Trans. Inf. Theory 22, $422-431$. 